\title{
Minimización de la distorsión armónica de una modulación PWM con algoritmos genéticos
}

\author{
Andrés Fernando Lizcano Villamizar \\ Ing. Electrónico, Universidad de Pamplona \\ Estudiante Maestría, Investigador Grupo A\&C, \\ Universidad de Pamplona \\ Pamplona, Colombia \\ aflizcano@gmail.com
}

\author{
Jorge Luis Díaz Rodríguez \\ M.Sc. Control Automático, UCLV \\ Docente Tiempo Completo, Investigador Grupo A\&C, \\ Universidad de Pamplona \\ Pamplona, Colombia \\ jdiazcu@gmail.com
}

\author{
Aldo Pardo García \\ PhD. Ciencias Técnicas, IMAA \\ Docente Tiempo Completo, Director Grupo A\&C, \\ Universidad de Pamplona \\ Pamplona, Colombia \\ apardo13@hotmail.com
}

\begin{abstract}
Resumen-En este trabajo se describe una nueva estrategia de modulación por anchura de pulso (PWM, Pulse Width Modulation) optimizada por el método directo y la aplicación de Algoritmos Genéticos (GA, Genetic Algorithms) para la minimización del contenido armónico específicamente el quinto y séptimo armónico del contenido total, basado en la minimización de la distorsión armónica total (THD, Total Harmonic Distorsion). Se expone el desarrollo del método, las pautas necesarias incluido el desarrollo del algoritmo de la estrategia dentro del Procesador Digital de Señales (DSP, Digital Signal Processor) y visualizado los resultados obtenidos.
\end{abstract}

Palabras clave- Modulación por ancho de pulso, eliminación selectiva de armónicos, algoritmos genéticos, distorsión armónica, Procesador Digital de Señales, Transformada Rápida de Fourier.

\begin{abstract}
This paper describes a new strategy for pulse width modulation (PWM) optimized by the direct method and the application of genetic algorithms (GA) to minimize the harmonic content specifically the fifth and seventh harmonic total content, based on minimizing total harmonic distortion (THD). We describe the development of the method, the guidelines to take into account including the development of the algorithm, the strategy within the Digital Signal Processor (DSP), and visualizing the results.
\end{abstract}

Keywords - Pulse Width Modulation, Selective Harmonic Elimination, Genetic Algorithms, Harmonic Distortion, Digital Signal Processing, Fast Fourier Trasform.

\section{INTRODUCCIÓN}

La gran evolución de los procesos industriales soportados por máquinas eléctricas de corriente alterna ha aumentado la demanda de estrategias altamente confiables para el control y funcionamiento óptimo de las mismas, por eso, se hace necesario estudiar e investigar diferentes procesos que permitan suplir dichas necesidades, las cuales dan origen al desarrollo de aplicaciones como la presentada en este trabajo [1-4]. Una de las grandes motivaciones es la disminución del consumo energético de los dispositivos, además de esto la reducción en las pérdidas de conmutación y la reducción del contenido de armónicos.

La utilización de técnicas de inteligencia artificial, como lo son los Algoritmos Genéticos, establece una nueva herramienta para la optimización, a pesar de su relativa actualidad no resulta complejo su implementación, por lo que constituye una herramientas eficiente y atractiva a la hora de solucionar un problema de optimización [5].

Al revisar la literatura sobre el tema se puede verificar que la aplicación de algoritmos genéticos en los accionamientos eléctricos, para optimizar la modulación PWM en los inversores de frecuencias, data de la última década, dentro de los primeros trabajos sobre el tema se destaca el de los autores K. L. Shi y H. Li (2003) [6], en este trabajo se aplica un algoritmo genético para optimizar la modulación PWM de un inversor, con el que se obtiene resultados superiores a la modulación sinusoidal PWM (SPWM) triangular estándar y a la modulación PWM aleatoria. Sin embargo, ambas modulaciones no representan un punto de partida adecuado, ya que se pueden agregar otros parámetros a la modulación SPWM triangular como la asimetría $[7,8]$ o utilizar otras modulaciones PWM que garanticen menor contenido de distorsión armónica [9]. También se pueden utilizar 
otros criterios de optimización armónica como es el contenido armónico del $5^{\text {to }}$ y $7^{\text {to }}$ armónicos, que resultan los armónicos más representativos por ser los de menor orden producidos por el inversor el cual no genera armónicos pares y los armónicos de $3^{\text {er }}$ orden se suprimen con una conexión adecuada del motor.

\section{MODULACIÓN PWM OPTIMIZADA}

El auge de los dispositivos digitales programables, tales como los Procesadores Digitales de Señales o DSP, han permitido que estas técnicas PWM puedan ser implementadas de una manera cómoda y eficiente y permitir así la gran evolución de las estrategias de modulación incluida la disminución de costos para su desarrollo [4, 9].

Para generar una modulación PWM optimizada se necesita de un determinado conjunto de ángulos (posiciones) de conmutación que se determinan mwdiante métodos numéricos y computacionales. En la Fig. 1 se muestra una señal PWM optimizada por un método directo de 3 pulsos $[4,9]$.

La modulación PWM optimizada presenta la ventaja de no usar una señal portadora, como el caso de la modulación PWM sinusoidal (SPWM), ya que el algoritmo genera internamente los ángulos de conmutación desde el dispositivo por medio de la reproducción de patrones de onda debidamente conformados, como los de la Fig. 1.

FIG. 1. PWM OPTIMIZADA POR EL MÉTODO DIRECTO

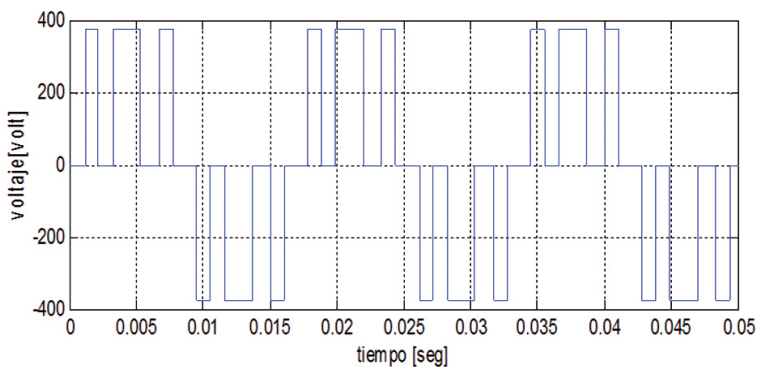

Fuente: Autor del proyecto

A continuación se comenta el procedimiento utilizado para generar la PWM optimizada, a partir de las expresiones de desarrollo del método planteado $[4,9]$.

A partir de la expresión de la referencia sinusoidal:

$$
u=V_{m} \operatorname{sen} \omega t
$$

Donde:

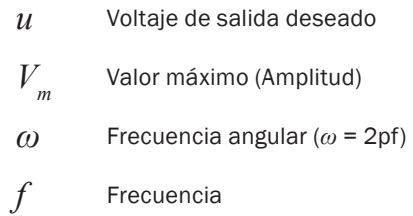

El área de un semiperíodo de $(0$ a $\pi / \omega)$

$A_{\text {seno }}=\int_{0}^{\pi / \omega} V_{m} \operatorname{sen} \omega t d t=-\left.\frac{V_{m}}{\omega} \cos \omega t\right|_{0} ^{\pi / \omega}=\frac{2 V_{m}}{\omega}$

Esto se puede dividir en $\mathrm{np}$ intervalos de tiempo regulares como se muestra a continuación $[4,9]$ :

$$
\left.\begin{array}{c}
t_{0}=0 \\
t_{1}=\frac{\pi}{n_{p} \omega} \\
\ldots \ldots \ldots \ldots \ldots . . . . \\
t_{n}=\frac{n_{p} \pi}{n_{p} \omega}=\frac{\pi}{\omega}
\end{array}\right\} \Rightarrow t_{i}=\frac{i \pi}{n_{p} \omega} \quad i=0,1,2, \cdots, n_{p}
$$

El área de cada uno de los intervalos es:

$$
\begin{aligned}
& A_{i}=\int_{t_{i-1}}^{t_{i}} V_{m} \operatorname{sen} \omega t d t=-\frac{V_{m}}{\omega} \cos \omega t||_{t_{i-1}}^{t_{i}} \\
& A_{i}=\frac{V_{m}}{\omega}\left(\cos \omega t_{i-1}-\cos \omega t_{i}\right)
\end{aligned}
$$

Conocida el área de cada uno de los intervalos y fijado el valor máximo de la onda PWM igual a la amplitud de la señal sinusoidal, se puede determinar el ancho de los pulsos.

$$
t p_{i}=\frac{A_{i}}{V_{m}}
$$

Finalmente, para la conformación de la señal PMW bajo el criterio de optimización antes expuesto, se ubican cada uno de los pulsos obtenidos en el centro de cada uno de los intervalos, se expresa matemáticamente este conjunto de pulsos en un plano cartesiano $x y$, donde el eje $x$ corresponde con el tiempo $y$ el eje $y$ con la amplitud [4], como se muestra:

$$
\begin{aligned}
& \text { Pulso }_{i}=\{\left\{\begin{array}{l}
(x, y) / \frac{x_{i-1}+x_{i}}{2}-\frac{t p_{i}}{2} \leq x \leq \frac{x_{i-1}+x_{i}}{2}+\frac{t p_{i}}{2}
\end{array}\right\} \\
& \cdots 0 \leq y \leq V_{m} \\
& \text { siendo } i=0,1,2, \cdots, n_{p}
\end{aligned}
$$




\section{A. Simulación del sistema}

Para validar la metodología anterior y obtener así la modulación PWM que se va a optimizar se desarrolla un modelo con el uso del Simulink ${ }^{\circledR}$ del Matlab ${ }^{\circledR}$ encargado de generar la modulación PWM optimizada por fase (Fig. 2).

Con este modelo anterior se genera un bloque que posee las siguientes entradas: la amplitud $(V)$, la frecuencia $(\omega)$, el número de pulsos (Np) y la asimetría de la señal portadora triangular (Pp), como se muestra en la Fig. 3, esta varía en un rango de 0 a 1 y modifica la posición de los pulsos.

FIG. 2. MODELO DE LA MODULACIÓN PWM OPTIMIZADA

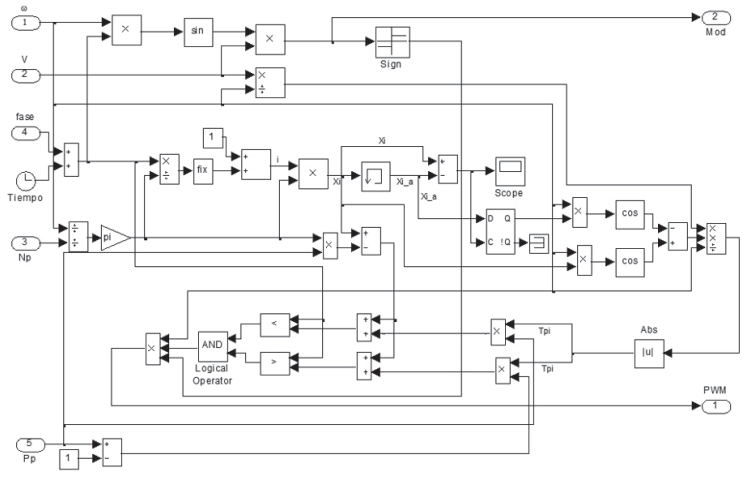

FIG. 3. VALORES DE LA POSICIÓN DEL PULSO


En la Fig. 4 se muestra el programa de simulación correspondiente a la descripción de la modulación PWM desarrollada y el respectivo cálculo del contenido de armónicos (THD) total y del $5^{\text {to }}$ y $7^{\mathrm{mo}}$ armónicos tanto de manera gráfica como de manera numérica.
La Fig. 5 muestra el resultado de la modulación para una de las fases del inversor y la siguiente muestra el espectro de armónicas para una frecuencia fundamental de $60 \mathrm{~Hz}$, un número de pulsos $\mathrm{Np}=33$ y posición del pulso de $\mathrm{Pp}=0.45$ asimétrico con respecto al eje de referencia central.

La Fig. 6 muestra la aplicación de la transformada rápida de Fourier (FFT) a la modulación.

Los parámetros de entrada están colocados de forma aleatoria, lo que no asegura que la modulación PWM obtenida sea la mejor, como se puede observar el espectro de frecuencia, inclusive tiene contenido armónico de componente directa, lo cual no es deseado. Pero se observa que el quinto y séptimo armónico son bastante bajos en amplitud lo que hace que no sea del todo mala la respuesta obtenida. De forma numérica obtenemos el resultado de una distorsión armónica total correspondiente al quinto y séptimo armónico igual al 23\%, la cual es adecuada, pero que se puede disminuir aún más con un proceso de optimización.

FIG. 4. MODELO EN SIMULINK® PARA OBTENER LA DISTORSIÓN ARMÓNICA

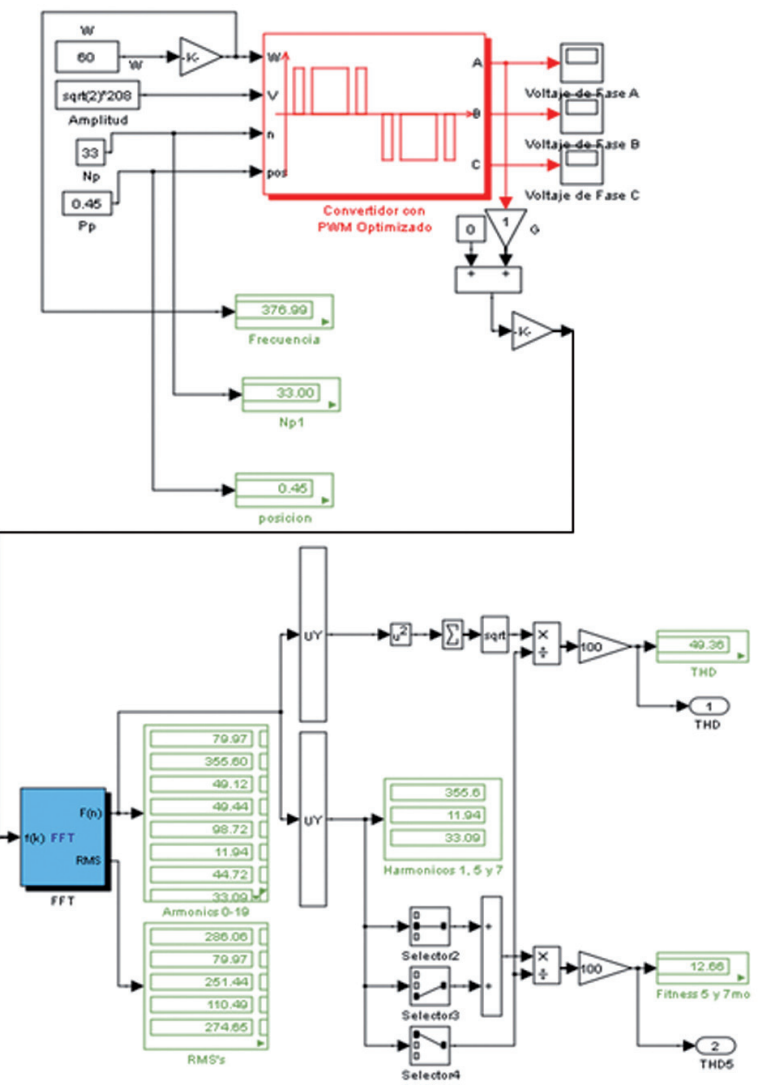

Fuente: Autor del proyecto. 
FIG. 5. MODULACIÓN PWM (NP=33, PP=0.45)

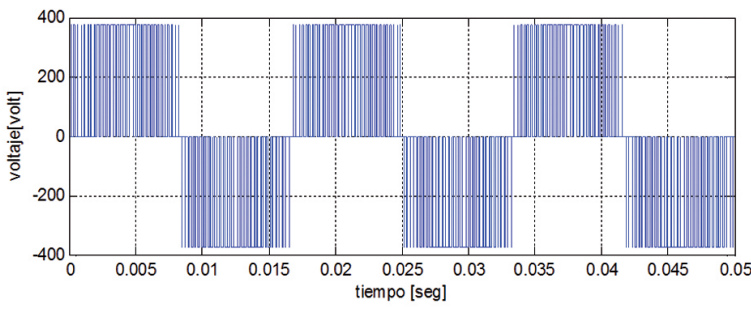

Fuente: Autor del proyecto

FIG 6. ANÁLISIS FFT DE LA SEÑAL PWM



Fuente: Autor del proyecto

\section{OPTIMIZACIÓN DE LA MODULACIÓN PWM CON ALGORTIMOS GENÉTICOS}

El propósito de la aplicación de Algoritmos Genéticos es la optimización de la modulación PWM, esto se logra a través de la creación de un conjunto de individuos (diferentes valores de frecuencias, número de pulsos y posición del pulso) que representan las posibles soluciones al problema en particular que se quiere optimizar y buscar la menor distorsión armónica del sistema, lo que hace que la función objetivo (fitness) a optimizar sea la distorsión armónica equivalente al $5^{\text {to }}$ y $7^{\text {mo }}$ armónicos de la señal PWM de salida. Cada uno de los valores del THD es sustraído de la constante $\alpha$ que corresponde al máximo valor de distorsión permitido estandarizado dentro del algoritmo genético. La función objetivo es:

$$
F=\alpha-\frac{\sqrt{v_{5}^{2}+v_{7}^{2}}}{v_{1}}
$$

\section{Donde:}

$F \quad$ Función objetivo (fitness).

$\alpha \quad$ Máximo valor del fitness estipulado.

$v_{5} \quad$ Magnitud del voltaje del $5^{\text {to }}$ armónico.

$v_{7} \quad$ Magnitud del voltaje del $7^{\mathrm{mo}}$ armónico.

$v_{1} \quad$ Magnitud del voltaje del armónico fund.

En la ecuación anterior se toman solamente las magnitudes del $5^{\text {to }}$ y $7^{\text {mo }}$ armónico, ya que por la forma de la señal sólo se deben de presentar armónicos impares y el tercer armónico se elimina posteriormente por la conexión en triángulo del motor asíncrono trifásico. Los armónicos más representativos por su bajo orden resultarían el $5^{\text {to }} \mathrm{y}$ $7^{\mathrm{mo}}$ armónico, los otros armónicos por estar alejados de la frecuencia fundamental influyen mucho menos en el funcionamiento de la máquina.

En la Fig. 7 se muestra el diagrama en bloque de la estructura del Algoritmo Genético implementado [7]. Como se observa en esta figura el primer paso que se realiza es crear una población inicial que hace referencia a las posibles soluciones al problema, después se hace la evaluación de la función objetivo y sólo los individuos más aptos pasarán a la siguiente etapa, en la cual se les aplica la mutación y cruce para generar nuevos individuos con mejores características que serán evaluados de nuevo. Así sucesivamente se repite el procedimiento hasta encontrar el mejor resultado posible en 100 generaciones.

FIG. 7. DIAGRAMA DEL ALGORITMO GENÉTICO

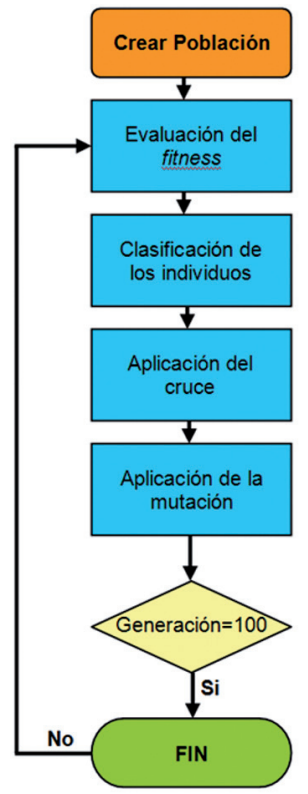

Fuente: Autor del proyecto.

El algoritmo genético puede detenerse de dos maneras, la primera es si cumple la optimización deseada y la segunda si termina de ejecutar el número de generaciones estipuladas.

Una de las grandes ventajas de aplicar un algoritmo genético es que este asegura que así no se llegue a encontrar una respuesta de error nulo, logrará al terminar el proceso el individuo más apto para conseguir el menor error posible. Como 
se menciona anteriormente los algoritmos genéticos parten del hecho de soluciones aleatorias, en nuestro caso de estudio se decide tomar un rango de individuos de 100 de acuerdo que a la disposición de hardware donde se aplica la herramienta la cual esta restringida a esta cantidad de posibles soluciones, por lo cual no nos permite establece un rango mayor de individuos.

El algoritmo es desarrollado en la herramienta de Simulink ${ }^{\circledR}$ de Matlab $^{\circledR}$, donde actúa de manera interactiva con los datos generados por el modelo del inversor trifásico, de esta manera se aplica el algoritmo y de forma inmediata se van generando las soluciones óptimas para la disminución de la distorsión armónica.

Los algoritmos genéticos, poseen una característica común con las diversas técnicas de inteligencia artificial, es el tiempo significativo o considerable que toma para ejecutarse y encontrar posibles soluciones, para nuestro caso y contando con el número de individuos se toma de 4 a 6 horas en ejecutarse en su totalidad, la idea de tomar un algoritmo genético como herramienta de búsqueda de soluciones se hace para optimizar al máximo el resultado.

Para cada una de las frecuencias el algoritmo evalúa 100 diferentes y posibles soluciones (individuos), la estadística se genera cuando el algoritmo clasifica cada una de las soluciones y coloca en primera posición la respuesta más clara y de mejor resultado (óptima) para obtener una disminución significativa de la distorsión armónica.

\section{A. Resultados}

En la tabla I se muestran los resultados para algunas de las frecuencias cercanas a $60 \mathrm{~Hz}$, donde se presentan los parámetros más aptos para la implementación de la modulación.

Tabla I:

optimización para frecuencias cercanas a la frecuencia nominal $(60 \mathrm{~Hz})$

\begin{tabular}{|c|c|c|c|}
\hline $\begin{array}{c}\text { Frecuencia } \\
(\mathrm{Hz})\end{array}$ & $\begin{array}{c}\text { No. pulsos } \\
(\mathrm{Np})\end{array}$ & $\begin{array}{c}\text { Posición } \\
(0<\mathrm{Pp} \leq 1)\end{array}$ & $\begin{array}{c}\text { THD } 5^{\mathrm{to}}+7^{\mathrm{mo}} \\
(\%)\end{array}$ \\
\hline 58 & 129 & 0.9661 & 10.9916 \\
\hline 59 & 111 & 0.9520 & 5.2787 \\
\hline 60 & 15 & 0.3392 & 6.2368 \\
\hline
\end{tabular}

Fuente: Autor del proyecto.
Para mostrar el resultado del algoritmo mediante el cual se obtiene el individuo óptimo y se consigue la menor distorsión posible, se muestra en la fig. 8 la transformada rápida de Fourier (FFT) de la señal PWM optimizada para $60 \mathrm{~Hz}$.

FIG. 8. ESPECTRO DE LA SEÑAL PWM PARA $60 \mathrm{HZ.}$

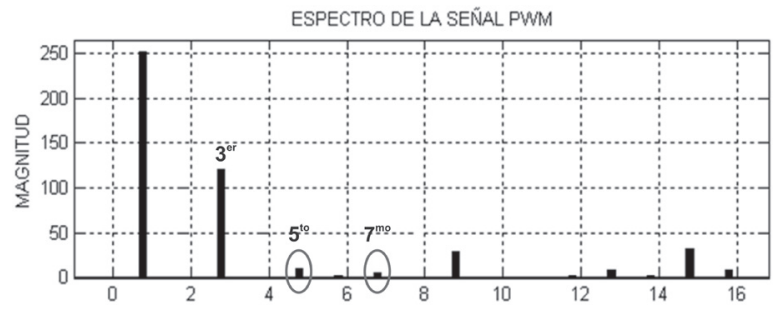

Fuente: Autor del proyecto.

En la figura anterior se puede observar la disminución del $5^{\text {to }}$ y $7^{\text {mo }}$ armónicos de una manera considerable, la no existencia de armónicos pares y el aumento del $3^{\text {er }}$ armónico que no reviste importancia ya que la conexión en triángulo del motor trifásico no permite que circulen estos hacia la fuente de alimentación. Al aplicar la modulacion PWM optimizada al motor podemos conseguir la onda de corriente y su espectro de frecuencia (fig. 9) donde se obtiene un porcentaje de distorsión armónica inferior al $1 \%$.

FIG. 9. ESPECTRO DE LA CORRIENTE PARA $60 \mathrm{HZ}$.



Fuente: Autor del proyecto.

\section{IMPLEMENTACIÓN DE LA ESTRATEGIA EN EL DSP 56F801}

Como dispositivo de control se utilizó un procesador digital de señales DSP 56F801 de FREESCALE ${ }^{\circledR}$ de punto flotante, ya que en comparación con otro dispositivo, como un microcontrolador, posee mayor más capacidad de cálculo y almacenamiento de memoria; para la implementación de la estrategia se optó por realizar los cálculos dentro del mismo algoritmo del DSP, la fig. 10 muestra un diagrama en bloques del sistema implementado. 
FIG. 10. ESQUEMA DE LA IMPLEMENTACIÓN DE LA ESTRATEGIA DE MODULACIÓN EN EL DSP.

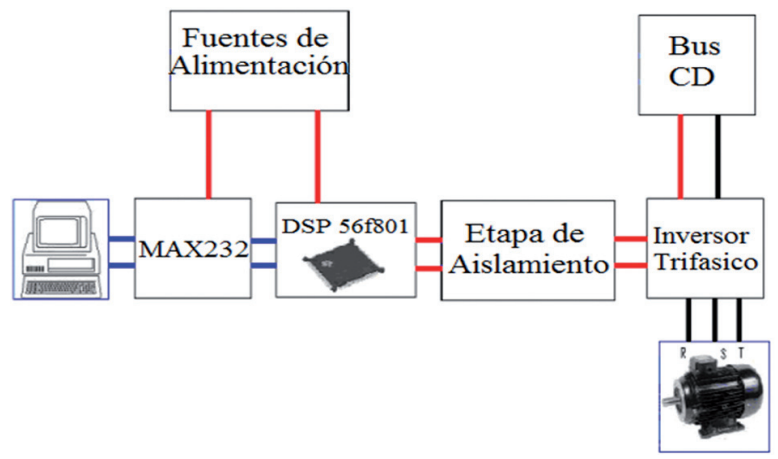

Fuente: Autor del proyecto.

Este diagrama anterior muestra que los datos, como son el número de pulsos, la frecuencia de trabajo y la posición del pulso, son enviadas desde una interfaz gráfica del computador hasta el DSP que captura estos datos, los procesa y genera las señales de control que irán hacia el inversor al cual se conecta el motor trifásico a controlar.

El sistema de control tal como muestra la fig. 11 está compuesto por el DSP, el programador del DSP, el circuito de adaptación de niveles TTL a RS232 (construido a partir de un MAX232) y el osciloscopio para registrar las formas de ondas.

FIG. 11. SISTEMA DE CONTROL CON EL PROCESADOR DIGITAL DE SEÑALES DSP 56F801.

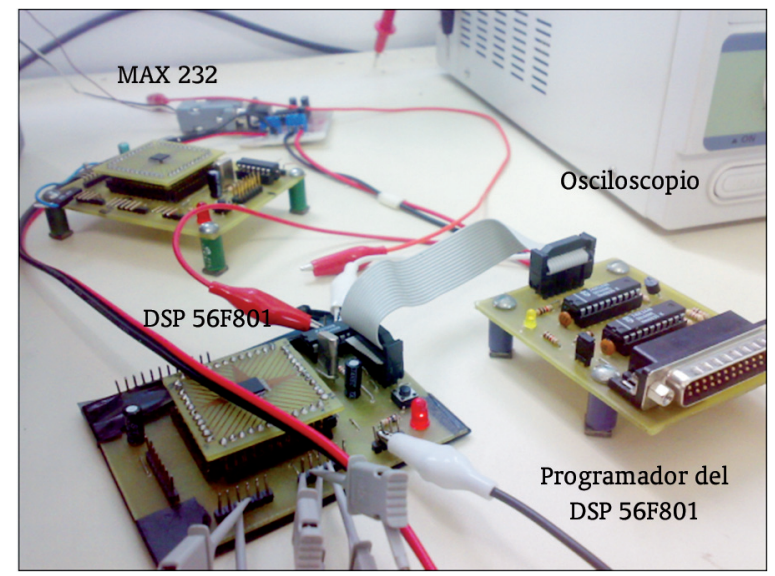

Fuente: Autor del proyecto.

El sistema empieza a funcionar cuando a través de la interfaz gráfica se envían los datos hacia el Procesador que almacena las formas de ondas PWM resultado del proceso de optimización y realiza la conformación de la modulación PWM por fase cuyas salidas son visualizadas en el osciloscopio (ver figs. 12 al 14).
En la fig. 12 se muestra las 6 señales de control que van hacia el inversor, 3 señales de activación de la parte alta del puente y 3 señales de activación de la parte baja, desfasadas $120^{\circ}$ entre sí.

FIG. 12. SEÑALES DE LA MODULACIÓN PWM OPTIMIZADA GENERADAS CON EL DSP PARA UN NP=15.



Fuente: Autor del proyecto.

En la figs. 13, 14 y 15 se muestran el voltaje de fase $(A B)$, el voltaje de línea $(A)$ y el espectro armónico generado a la salida del inversor.

FIG. 13. VOLTAJE ENTRE LAS FASES (AB).

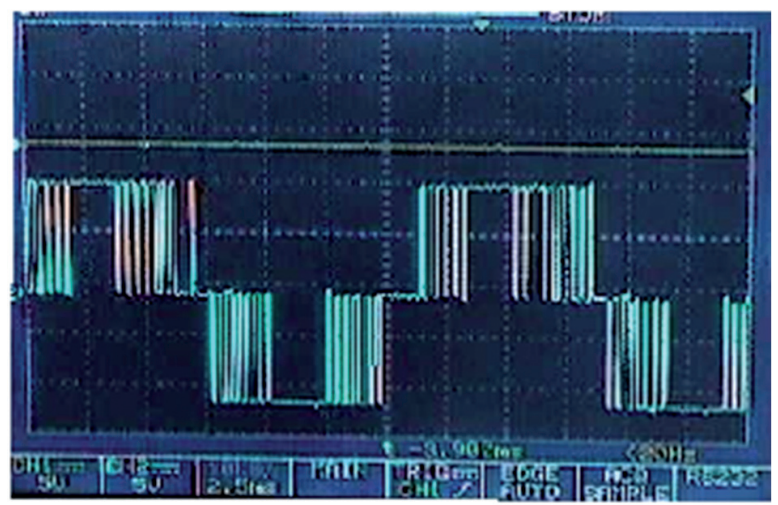

Fuente: Autor del proyecto.

FIG. 14. VOLTAJE DE LÍNEA (A).

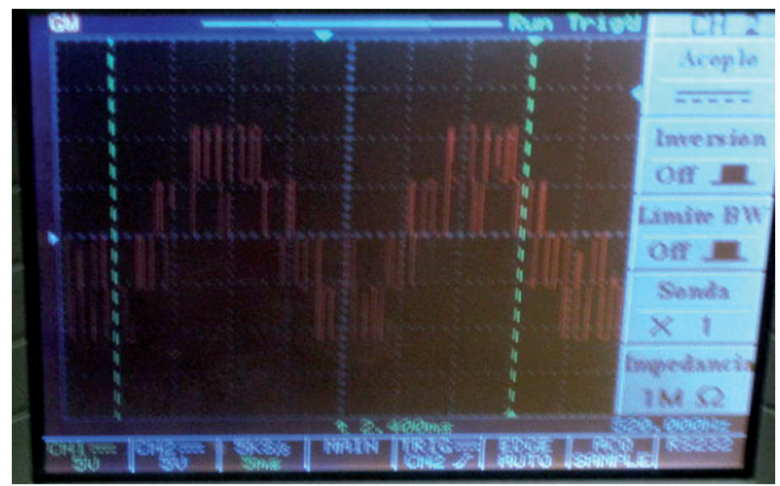

Fuente: Autor del proyecto. 
FIG. 15. ESPECTRO ARMÓNICO.

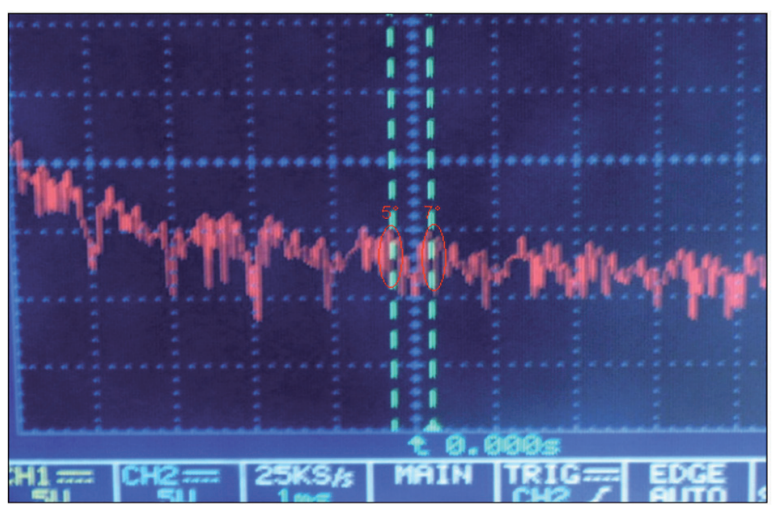

Fuente: Autor del proyecto.

\section{MEJORAMIENTO DE LA CALIDAD DE LA ENERGÍA}

Al diseñar una estrategia PWM que brinde las mejores condiciones en cuanto a disminución de la distorsión armónica se asegura la entrega de una señal adecuada la cual brindará las mejores características de trabajo a la máquina eléctrica o dispositivo que se conecte, lo que se contribuye directamente a la calidad de la energía [10].

Con la motivación anterior, la estrategia PWM que se plantea en este trabajo aborda a lo largo de su desarrollo a un objetivo explicito: la optimización de la distorsión armónica.

El desarrollo de esta estrategia de modulación PWM con bajo contenido de armónicos genera una gran confiabilidad a la hora de evaluar la calidad de energía entregada por el inversor, permitiendo establecer con esta modulación una magnitud de eficiencia mayor en función de la menor distorsión armónica generada.

\section{CONCLUSIONES}

Los algoritmos genéticos son estrategias eficientes y robustas para la solución de problemas no triviales que contribuyen en gran medida a la optimización de diferentes procesos donde son requeridos. Gracias a la utilización de estas técnicas se han podido encontrar las mejores condiciones de trabajo para los diferentes barridos de frecuencia donde se trabaja la modulación PWM y llega a inferirse que la modulación obtenida es óptima posible. Al optimizar la señal de modulación PWM directamente se optimiza la distorsión armónica de la corriente del motor en un porcentaje suficientemente bueno para la máquina $(<1 \%)$.

Al finalizar el proceso de selección mediante Algoritmos Genéticos, se establecieron los individuos más aptos para encontrar la menor distorsión armónica posible entregada por el inversor a nivel del quinto y séptimo armónico.

El desarrollo del Algoritmo Genético se hace de manera offline debido a que estas estrategias de inteligencia artificial se caracterizan por su extenso tiempo de generación y alto consumo de recursos de memoria, por lo cual los individuos más aptos son obtenidos a nivel de simulación del algoritmo y entonces programados al DSP.

Finalmente, una vez conducido el análisis de los resultados teóricos y prácticos de optimización de la modulación PWM, el trabajo aporta una minimización del contenido armónico partiendo de onda PWM optimizada, desarrollada por los autores, como punto de partida del procedimiento de optimización, y utilizando un criterio de optimización (fitness) basado en el contenido del $5^{\text {to }}$ y $7^{\text {mo }}$ armónicos que garantiza la optimización con menos esfuerzo computacional que sí se calcula con base en el valor de la distorsión armónica total (THD), para su determinación se requiere del conocimiento de cada armónico individual. La onda PWM optimizada garantiza el funcionamiento eficiente del conjunto inversor - motor, ya que minimiza el $5^{\text {to }}$ y $7^{\text {mo }}$ armónicos, que son los máximos responsables de los pares de frenado parásitos por ser de secuencia negativa y también logra reducir la cantidad de pulsos ( $\mathrm{Np}$ ) de la modulación PWM disminuyendo de esta forma las pérdidas por conmutación en el inversor.

\section{REFERENCIAS}

[1] M. H. Rashid, Power Electronics Handbook, Second Edition: Devices, Circuits and Applications". Academic Press; 2 Ed., 2007.

[2] M. H. Rashid, Power Electronics. Prentice Hall, India, 2008.

[3] N. Mohan, T. M. Undeland y W. P. Robbins, Electrónica de Potencia. Convertidores, aplicaciones y diseño, McGraw Hill Interamericana, 3ra Edición, México D.F., 2009. 
[4] A. Pardo y J. L. Díaz, Aplicaciones de los convertidores de frecuencia, Estrategias PWM, Editorial Java E. U., Colombia, 2004.

[5] D. E. Goldberg, Genetic algorithms in search, optimization \& machine learning, Addison-Wesley, Reading, 1989.

[6] K. L. Shi and H. Li, "Optimized random PWM strategy based on Genetic algorithms", IEEE Trans. Mag. Vol. 33, pp. 07-11, 2003.

[7] B. Justus Rabi, "Minimization of Harmonics in PWM inverters based on Genetic Algorithms". Journal of Applied Sciences 6 (9): 2056-2059, 2006.

[8] N. Tutkun, "A new modulation approach to decrease total harmonic distortion of the SPWM voltage waveform using genetic optimization technique". International Conference on Renewable Energies and Power Quality (ICREPQ'08), Palacio de la Magdalena de Santander, 12-14 de Marzo de 2008.

[9] J. L. Díaz, A. Pardo y E. Y. Ríos. “Implementación de una modulación PWM optimizada para el control de un motor trifásico de inducción usando la tarjeta DSP TMS320C6416 DSK", Revista Colombiana de Tecnologías de Avanzada, Vol. 1, No. 13, 2009.

[10] G. Carrillo, et al., "Caracterización, compensación y medición de un sistema de distribución de energía eléctrica con alta polución armónica”. Primer Simposio Internacional sobre Calidad de la Energía Eléctrica, Bogotá, Noviembre 2001. 